\title{
Pandemia de COVID-19 e ocupação no mercado de trabalho: o caso da região Nordeste do Brasil*
}

\author{
Pedro Rodrigues Oliveira ${ }^{\dagger}$ \\ Stéffany Costa Jardim \\ Evandro Camargos Teixeira ${ }^{\S}$
}

\begin{abstract}
Resumo
Este trabalho tem como objetivo central analisar o impacto da pandemia da COVID-19 sobre os níveis de ocupação nos setores da indústria, comércio, construção e transporte na região Nordeste do Brasil através da estimação de quatro modelos Probit. Com exceção do setor de construção, todos os demais setores considerados foram impactados negativamente pela pandemia. Especificamente, a presença da COVID-19 no $1^{\circ}$ trimestre de 2020 reduziu a probabilidade de ocupação em 0,142 p.p. na indústria, 0,423 p.p. no setor de comércio e 0,054 p.p. no segmento de transporte.
\end{abstract}

Palavras-chave: COVID-19, Ocupação, Nordeste, Brasil.

JEL: J01, J00

\section{Introdução}

A humanidade enfrenta atualmente uma das maiores crises de saúde pública já existentes na história do planeta, a pandemia do COVID-19. Esta nomenclatura foi atribuída pela Organização Mundial de Saúde (OMS) para a doença causada pelo novo coronavírus ou SARS-CoV-2, que se caracteriza por ser altamente contagiosa e de dimensões ainda desconhecidas. O grande desafio imposto pelo novo coronavírus está relacionado ao fato de não haver um tratamento farmacêutico, o que levam os governos a praticarem medidas alternativas para conter a disseminação do vírus. As medidas apontadas - até então por cientistas e formuladores de políticas públicas como mais seguras e defendidas pela

\footnotetext{
*Agradecemos os comentários e sugestões

${ }^{\dagger}$ Graduando em Economia, Universidade Federal de Viçosa, Departamento de Economia, Viçosa, Brasil, E-mail: pedro.oliveira@ufv.br, ORCID:https://orcid.org/0000-0001-6630-9666

${ }_{\ddagger}^{\ddagger}$ Graduanda em Economia, Universidade Federal de Viçosa, Departamento de Economia, Viçosa, Brasil, E-mail: steffany.jardim@ufv.br, ORCID:https://orcid.org/0000-0002-7561-6606

§Professor Associado I, Universidade Federal de Viçosa, Departamento de Economia, Viçosa, Brasil, Email: evandro.teixeira@ufv.br, ORCID:https://orcid.org/0000-0002-6470-2103
} 
do Brasil

OMS são representadas pelo distanciamento social, isolamento e quarentena. Tais ações, adotadas a fim de impedir um colapso no sistema de saúde e grande letalidade advinda da contaminação, implicam em redução do nível de atividade econômica.

Nesse sentido, entre outras consequências, o impacto sobre as atividades produtivas tem elevado a quantidade de falências de empresas com efeito significativo sobre o nível de emprego, oriundo da demissão em massa de trabalhadores. Para o Brasil, o Instituto Brasileiro de Economia da Fundação Getúlio Vargas (Ibre/FGV) prevê uma taxa de desemprego de 17,8\% para o ano de 2020, sendo, que em 2019, essa ficou em 11,9\%. Além disso, a Organização Internacional do Trabalho (OIT) aponta que quase 25 milhões de empregos podem ser perdidos no mundo devido a COVID-19.

Ao analisar os efeitos sobre a produção, uma pesquisa divulgada pelo Instituto Brasileiro de Economia da Fundação Getúlio Vargas (Ibre/FGV) aponta que já em março de 2020, o setor industrial se mostrou como o mais afetado, onde $43 \%$ das empresas relataram já enfrentarem os impactos negativos da pandemia de COVID-19; os setores de serviços e comércio aparecem em seguida como mais impactados, com 35,4\% e 30,2\%, respectivamente. Além disso, dados do Instituto Brasileiro de Geografia e Estatística (IBGE) mostram que o segmento de transporte, armazenagem e correio já observou no $1^{\circ}$ trimestre de 2020 redução de 2,4\% no produto em relação ao último trimestre de 2019.

Cabe ainda salientar que a crise causada pela pandemia interage com aspectos préexistentes dos países em que o vírus se dissemina. No Brasil, observa-se que o país já enfrentava uma realidade de recessão, seguida por dificuldade de recuperação na economia desde 2014. O Comitê de Datação de Ciclos Econômicos (CODACE) associado à Fundação Getúlio Vargas (FGV) identificou que o país enfrentou uma recessão de 33 meses, entre abril de 2014 e dezembro de 2016. Após este grande período de recessão, a economia brasileira permaneceu estagnada, com crescimento em torno de $1 \%$, apresentando um pico no ciclo de negócios no $4^{\circ}$ trimestre de 2019. Segundo o Comitê, o país novamente voltou a apresentar um quadro recessivo a partir do $1^{\circ}$ trimestre de 2020.

O impacto em termos produtivos no mercado de trabalho é direto. Nesse sentido, Lameiras et. al (2019) observaram que no ano de 2019, apesar de alguns indicativos favoráveis em relação a geração de empregos, o mercado de trabalho brasileiro seguia bastante deteriorado, com elevado contingente de desocupados e subocupados. Os autores destacaram ainda que o país apresentava uma quantidade crescente de desempregados, que estavam nessa situação a mais de dois anos. Como prova disso, o referido contingente avançou de $17,4 \%$ no $1^{\circ}$ trimestre de 2015 para 24,8\% no mesmo período em 2019.

Além da realidade de lenta recuperação econômica do país, constata-se elevada disparidade socioeconômica e de condições de enfrentamento à pandemia entre as regiões brasileiras, cuja precariedade mais elevada encontra-se no Nordeste. De acordo com o Índice Firjan de Desenvolvimento Municipal (IFDM), em 2016, a região nordestina continha $68 \%$ dos municípios menos desenvolvidos do Brasil, situação vigente durante a última década. Tal condição histórica está relacionada a problemas crônicos de educação, 
saúde, segurança e infraestrutura básica. Nesse sentido, em 2016, a região representava $14,8 \%$ do total de brasileiros que não sabiam ler e escrever, percentual este quatro vezes mais elevado que o da região Sudeste. Em relação às condições de saúde, $24,4 \%$ dos municípios nordestinos possuíam nota baixa ou regular referente ao acesso a saúde. Adicionalmente, a região possuía o menor rendimento mensal per capita do país, equivalente a $\mathrm{R} \$ 1429,00$, e estava abaixo da média nacional de abastecimento de água, esgoto sanitário e coleta de lixo. Destaca-se ainda que os indicadores da região apresentaram evolução nas últimas décadas, mesmo que lentamente, mas tal movimento foi interrompido pela recessão econômica iniciada em 2014, o que acentuou as disparidades regionais brasileiras.

Corroborando a análise sobre a relativa vulnerabilidade da região Nordeste, em março de 2020, a Fundação Oswaldo Cruz (Fiocruz) apresentou um Relatório de análise do risco de disseminação da COVID-19 e avaliação de vulnerabilidade social nas microrregiões brasileiras. Foi possível constatar que aquelas com maior risco de transmissão sustentada no curto prazo e com maior vulnerabilidade social estavam concentradas no Nordeste, especificamente nos estados do Ceará, Paraíba e Bahia. Essa previsão é confirmada posteriormente, em maio do mesmo ano, quando a região apresentou cerca de $30 \%$ do total de casos oficiais de COVID-19 no país, sendo aquela com a maior taxa de crescimento proporcional. Além disso, a maioria dos estados nordestinos apresentava letalidade acima do que é observado em média no país. Marinelli et. al (2020) apontam que essas informações sugerem a falta de capacidade de atendimento adequado aos casos iniciais, além da baixa cobertura de testagem. Adicionalmente, a região possui as menores taxas de leitos de Unidade de Terapia Intensiva (UTI) e profissionais de saúde por 100 mil habitantes do país, o que pode tornar mais difícil o enfrentamento à pandemia.

Nesse sentido, o primeiro caso da COVID-19 na região Nordeste brasileira ocorreu no estado do Ceará, ainda em janeiro de 2020. Entretanto, o primeiro caso registrado nas estatísticas oficiais somente ocorreu no dia 6 de março no estado da Bahia, sendo seguido por registros nos estados de Alagoas e Pernambuco, no dia 12 de março. As primeiras medidas de contenção do que viria a se tornar uma pandemia na região foram o isolamento social e medidas de restrição à mobilidade, que começaram a ser tomadas por volta do dia 15 de março de forma não coordenada, isto é, estados e municípios delimitaram sua própria metodologia de contenção da disseminação do vírus (COMITÊ CIENTÍFICO CONSÓRCIO NORDESTE SUBCOMITÊ DE EPIDEMIOLOGIA, 2020).

Diante dos aspectos mencionados, justifica-se a importância da análise de como a COVID-19 pode impactar o nível de emprego na região Nordeste do Brasil, uma vez que a mesma se apresenta como a mais fragilizada em termos socioeconômicos e a crise causada pela pandemia pode ter efeitos significativos em termos de aprofundamento do desemprego na região. Dessa forma, o presente estudo tem como objetivo central analisar o efeito da pandemia do COVID-19 sobre a ocupação no mercado de trabalho da região Nordeste brasileira, levando em consideração os seguintes setores: i) indústria; ii) 

do Brasil

construção; iii) comércio e iv) transporte.

Tais setores, segundo Machado (2019), são aqueles, além da administração pública, com maior expressividade na ocupação dos postos de trabalho na região, de modo que estão entre os mais impactados no $1^{\circ}$ trimestre de 2020 em decorrência da pandemia, como descrito anteriormente. Ademais, eles compõem os grupamentos considerados pela Pesquisa Nacional por Amostra de Domicílios Contínua (PNADC), que é a fonte de dados utilizada no presente estudo. Nesse sentido, são utilizados dados do $1^{\circ}$ trimestre da supracitada pesquisa entre os anos de 2017 e 2020. Destaca-se ainda que não foram encontrados na literatura trabalhos que visassem analisar o efeito da pandemia da COVID-19 sobre o nível de emprego no Nordeste brasileiro, o que confere ao presente estudo ineditismo na referida análise.

Com esta finalidade, o trabalho se divide em quatro seções posteriores a esta. Inicialmente, realiza-se a revisão de literatura, que irá mapear os principais estudos que analisam os efeitos da COVID-19 sobre o mercado de trabalho em diversos países. Na terceira seção, apresenta-se a metodologia, com especificação do modelo econométrico a ser utilizado, bem como as variáveis e suas fontes. A penúltima seção trata dos resultados obtidos através das estimativas econométricas e uma análise detalhada da ocupação nos subsetores ao longo dos trimestres analisados. Por fim, apresenta-se as considerações finais.

\section{Revisão da Literatura}

Devido ao alarmante impacto da pandemia da COVID-19 sobre as vidas de bilhões de indivíduos ao redor do mundo, a análise da Economia é fundamental para que se possa compreender os efeitos da crise, planejar políticas públicas no intuito de que os mesmos sejam minimizados, além de traçar estratégias para a retomada das atividades produtivas em um futuro próximo.

Assim, Hevia e Neumeyer (2020), por exemplo, analisaram o impacto econômico da COVID-19 em todo o mundo. Os autores afirmam que a impossibilidade de isolar apenas os indivíduos infectados levou a uma política de distanciamento social, que impõe um custo econômico e social bastante oneroso. A combinação de políticas como distanciamento social, lockdown e quarentena implicam em redução ou até mesmo completa paralisação de diversas atividades produtivas e de consumo por determinado período de tempo, levando mercados ao colapso e potencialmente ocasionando demissão de milhões de pessoas.

Devido à restrição de mão-de-obra sofrida pelo setor produtivo e de circulação dos consumidores, Açikgöz e Günay (2020) discorrem que a pandemia afetará o mais básico equilíbrio entre a oferta e a demanda. Assim, os países entrarão em uma recessão encabeçada a priori pela queda de atividades, sobretudo dos setores de turismo e comércio

Econômica-Niterói, v. 22, n. 1, p. 9-30. Junho, 2020 
(imediatamente mais sensíveis à diminuição da circulação de pessoas) e, posteriormente, pela queda da atividade econômica de maneira generalizada. Logo, o desemprego e falências de empresas em massa serão fenômenos esperados.

Nesse sentido, de acordo com Yamin (2020), a classe trabalhadora mundial está sendo severamente impactada pela desaceleração repentina e inesperada da economia mundial, causando perda de emprego em níveis elevados em todos os países. Uma das razões citadas é o fato de que mais de quatro quintos da força de trabalho vive em países que sofreram severas restrições de distanciamento e situações de lockdown. Consequentemente, muitos países desenvolvidos já enfrentam taxa de desemprego de dois dígitos, levando as pessoas a procurarem por auxílios de desemprego. Resultados similares foram encontrados por Coates et al. (2020), Açikgöz e Günay (2020) e Dev e Sengupta (2020).

Como descrito, os impactos da crise serão grandes sobre a oferta de empregos, mas afetarão os setores de maneiras diferentes. Wagner (2020) pondera que do ponto de vista econômico a crise oriunda da COVID-19 pode ser dividida em três fases. A primeira fase ocorreu em dezembro de 2019 quando o vírus surgiu na cidade chinesa de Wuhan, onde observou-se crescente desvalorização de empresas do setor de transporte, dada a eminente restrição de circulação de pessoas. Na segunda fase, após a Organização Mundial da Saúde (OMS) conceder mais atenção a crise do vírus, observou-se valorização de empresas do setor de comunicação e tecnologia em virtude da predição da necessidade que o isolamento social traria quanto a possibilidade de novas formas de trabalho remoto. A terceira fase ocorreu em fevereiro de 2020 após a Itália implementar sua política nacional de isolamento. Nesta fase, a crise gerada pelo novo coronavírus se tornou evidente e amplamente divulgada, incorrendo em generalizada desvalorização de praticamente todos os setores econômicos devido ao temor dos investidores.

Makridis e Hartley (2020) consolidam os resultados de Wagner (2020) quanto à resposta da economia durante segunda fase da crise da COVID-19. Os autores estimaram o impacto das medidas de mitigação do novo coronavírus sobre a taxa de crescimento econômico dos EUA de 2020, analisando por setor industrial. Os autores concluíram que irão permanecer indústrias proporcionalmente ao seu nível de digitalização, usando como proxy os trabalhadores intensivos em tecnologia de informação em dada indústria. A justificativa é que estes não perderão seus empregos e continuarão trabalhando em suas casas e contribuindo para serviços que não dependem de interações pessoais.

Ao contrário dos trabalhadores intensivos em tecnologia de informação, que são menos sensíveis ao desemprego gerado pela pandemia, trabalhadores menos qualificados, especialmente em setores com baixo nível tecnológico, lidarão com outra realidade. Nesse sentido, Laing (2020) elaborou um estudo sobre o impacto da pandemia da COVID-19 sobre o setor de mineração. $\mathrm{O}$ autor discorre que o primeiro impacto da crise sobre o setor se deu de maneira indireta, através de diminuição da demanda advinda dos setores industrial e de construção, fortemente afetados pela crise. Devido a abrupta queda de demanda, a tendência é que haja crescimento da taxa de desemprego, especialmente em 

do Brasil

países pobres ou emergentes, dependentes das exportações de commodities.

Além dos trabalhadores menos qualificados, trabalhadores informais também enfrentarão a crise com maior severidade. Spurk e Straub (2020) afirmam que a depender do país em questão, a existência desse grupo de pessoas não é assegurada por seguro desemprego ou outro programa de financiamento, deixando-os desprotegidos. Assim, pode-se verificar aumento do desemprego em maior propensão em parcelas da população menos qualificadas e caracterizadas pela informalidade e, com o desemprego, haverá menor acesso aos serviços de saúde.

Nesse sentido, Gangopadhyaya e Garrett (2020) estimam o impacto que a crise causada pela pandemia do coronavírus causará sobre a taxa de desemprego e, consequentemente sobre o acesso dessas pessoas desempregadas aos planos de saúde e de auxílio financeiro à saúde. Com o desemprego, muitos trabalhadores irão perder uma importante fonte de renda, o que ocasionará na impossibilidade de que eles consigam arcar com o sistema de saúde. Além disso, muitos planos de saúde são providos por vínculos empregatícios. Logo, a crise no setor de saúde elevará as taxas de desemprego, que por sua vez prolongam a referida crise.

\section{Metodologia}

\subsection{Modelo econométrico}

O presente estudo tem como objetivo analisar em que magnitude a crise causada pela COVID-19 impacta a probabilidade de os indivíduos estarem ocupados em determinados setores produtivos na região Nordeste do país. Assim, tem-se uma variável dependente binária, pois os indivíduos podem estar ocupados ou não no $1^{\circ}$ trimestre em questão. Diante disso, a metodologia para a operacionalização do estudo contempla a estimação de um modelo Probit, onde a variável dependente é dicotômica, assumindo valores 0 ou 1, para indivíduos não ocupados ou ocupados, respectivamente.

De acordo com Davidson e Mackinnon (2004), o modelo Probit pressupõe em seu intervalo binário $(0,1)$ a manifestação observável de uma variável latente $\left(Y^{*}\right)$, sendo que seu sinal condicionará o valor da variável binária observada. Caso a variável latente assuma valor positivo, a variável binária assume valor igual a 1; caso a variável latente assuma valor negativo, a binária assume valor igual a 0 . Formalmente, tem-se a equação 1:

$$
Y_{i}=\left\{\begin{array}{lll}
1 & \text { se } & Y_{i}^{*} \geq 0 \\
0 & \text { se } & Y_{i}^{*}<0
\end{array}\right.
$$

Assim, é possível realizar a estimação, que calcula a probabilidade da variável $Y_{i}$

Econômica-Niterói, v. 22, n. 1, p. 9-30. Junho, 2020 
assumir va lor igual a 1, como descrito abaixo.

$$
\begin{array}{r}
\operatorname{Pr}\left(Y_{i}=1\right)=\operatorname{Pr}\left(Y_{i}^{*} \geq 0\right)=\operatorname{Pr}\left(X_{i} \beta+\mu_{i} \geq 0\right)= \\
=\operatorname{Pr}\left(\mu_{i} \geq-X_{i} \beta\right)=\Phi\left(X_{i} \beta\right)
\end{array}
$$

Onde $\Phi\left(X_{i} \beta\right)$ é a função de probabilidade cumulativa da distribuição normal padrão.

O presente estudo se vale de amplas evidências empíricas presentes na literatura, que utilizaram o referido modelo para estudos similares, como é o caso de Arruda et al. (2017), Guimarães e Santos (2009), Fleming e Kler (2016), Figueiredo et al. (2010) e Kingdon e Knight (2000). Cabe destacar que o modelo Probit limita-se a explicar eventos cuja função de distribuição de probabilidades seja do tipo normal. Desse modo, eventos que possuam outra função de distribuição dão origem a um modelo mal especificado com coeficientes estimados inconsistentemente.

De acordo com Arulampalam (1999), os impactos causados pelas variáveis explicativas sobre a ocupação, entretanto, não podem ser mensurados pela análise dos coeficientes estimados pelo modelo Probit. Para tal, é necessário estimar os efeitos marginais das variáveis explicativas.

Para $\operatorname{Pr}(Y=1)$, tem-se que o efeito marginal de uma variável $x$ contínua é dado pela equação 3:

$$
\frac{\partial \operatorname{Pr}(Y=1)}{\partial x_{k}}=\Phi\left(X^{\prime} \beta\right) \beta_{k}
$$

Para $\operatorname{Pr}(Y=0)$, tem-se que o efeito marginal de uma variável $x$ contínua é dado pela equação 4:

$$
\frac{\partial \operatorname{Pr}(Y=0)}{\partial x_{k}}=-\Phi\left(X^{\prime} \beta\right) \beta_{k}
$$

Assim, são estimados os efeitos marginais das variáveis explicativas, dentre elas a variável que mensura a influência da COVID-19 sobre a probabilidade de ocupação em determinados setores na região Nordeste brasileira. A partir dos resultados da estimação dos efeitos marginais, que sinalizam quais setores são impactados e em que magnitude pela COVID-19 na região Nordeste, será apresentada uma análise descritiva do nível de ocupação dos mesmos, incluindo seus subsetores, durante o período de análise do estudo.

\subsection{Fonte dos dados e descrição das variáveis}

Para realização desse estudo, foram utilizados dados da Pesquisa Nacional por Amostra de Domicílios Contínua (PNADC) referentes ao $1^{\circ}$ trimestre entre os anos de 2017 
do Brasil

e 2020 dos indivíduos residentes na região Nordeste do Brasil. As variáveis utilizadas tiveram como critério de seleção a literatura concernente ao tema e são apresentadas na Tabela 1.

Destaca-se que os dados da PNADC são coletados de acordo com um processo de amostragem complexa, conferindo às observações o caráter de estratificação, conglomeração, probabilidades desiguais de seleção e ajustes dos pesos para calibração. Dessa forma, é possível construir um recorte amostral verossímil à média populacional (SILVA; PESSOA; LILA, 2002).

Tabela 1 - Variáveis a serem utilizadas na estimação do modelo econométrico.

\begin{tabular}{|c|c|c|}
\hline Variável & Descrição & $\begin{array}{l}\text { Sinais } \\
\text { Esperados }\end{array}$ \\
\hline \multicolumn{3}{|c|}{ Variável Dependente } \\
\hline Ocupação & $\begin{array}{l}\text { Variável binária que considera indivíduos com idade entre } 14 \text { e } 65 \text { anos nos } \\
\text { setores produtivos analisados. Se eles estão ocupados, ela assume valor igual a } \\
\text { 1. Caso contrário, seu valor é igual a } 0 \text {. }\end{array}$ & \\
\hline \multicolumn{3}{|c|}{ Variáveis Explicativas } \\
\hline COVID-19 & $\begin{array}{l}\text { Variável binária que assume valor igual a } 1 \text { para o } 1^{\circ} \text { trimestre de } 2020 \text {, e } \\
\text { igual a } 0 \text { para os demais trimestres. }\end{array}$ & $(-)^{1}$ \\
\hline Gênero & $\begin{array}{l}\text { Variável binária que assume valor igual a } 1 \text { para indivíduos do gênero } \\
\text { masculino, e valor igual a } 0 \text { para indivíduos do sexo feminino. }\end{array}$ & $(+)^{2}$ \\
\hline Cor & $\begin{array}{l}\text { Variável binária que assume valor igual a } 1 \text { para indivíduos brancos e } \\
\text { amarelos, e igual a } 0 \text { caso contrário. }\end{array}$ & $(+)^{3}$ \\
\hline Idade & Variável contínua que contempla indivíduos na faixa etária de 14 a 65 anos. & $(+)^{4}$ \\
\hline $\begin{array}{l}\text { Pessoa de } \\
\text { Referência }\end{array}$ & $\begin{array}{l}\text { Variável binária que assume valor igual a } 1 \text { caso o indivíduo seja a pessoa de } \\
\text { referência do domicílio, e igual a } 0 \text { caso contrário }\end{array}$ & $(+)^{5}$ \\
\hline $\begin{array}{l}\text { Anos de } \\
\text { Estudo }\end{array}$ & Variável contínua que denota os anos de estudo dos indivíduos. & $(+)^{6}$ \\
\hline Urbano & $\begin{array}{l}\text { Variável binária que assume valor igual a } 1 \text { caso o indivíduo resida em meio } \\
\text { urbano, e valor igual a } 0 \text { caso contrário. }\end{array}$ & $(+)^{7}$ \\
\hline
\end{tabular}

Fonte: Elaboração própria

\footnotetext{
${ }^{1}$ De acordo com Gangopadhyaya e Garrett (2020) e Spurk e Straub (2020).

${ }^{2}$ De acordo com Coates et. al (2020).

${ }^{3}$ De acordo com Abramo (2006).

${ }^{4}$ De acordo com por Fernandes e Picchetti (1999).

${ }^{5}$ De acordo com Borges (2006).

${ }^{6}$ De acordo com Alves e Soares (1996).

${ }^{7}$ De acordo com por Monte e Penido (2008).
}

Econômica-Niterói, v. 22, n. 1, p. 9-30. Junho, 2020 


\section{Análise e discussão dos resultados}

Esta seção, subdividida em duas subseções, dedica-se a apresentar os principais resultados obtidos através da pesquisa. A primeira apresenta os resultados econométricos e a segunda analisa a evolução da ocupação na região Nordeste, por segmento setorial, ao longo dos trimestres analisados.

\subsection{Resultados econométricos}

Com intuito de verificar a magnitude do impacto da pandemia de COVID-19 sobre a ocupação em setores-chave da economia (indústria, comércio, construção e transporte) na região Nordeste, foram estimados quatro modelos Probit, vide Tabela 2. Destaca-se que os modelos foram estimados considerando-se erros padrão robustos com o objetivo de controlar a heterocedasticidade. Adicionalmente, todos os quatro modelos utilizados mostraram-se globalmente significativos pelo Teste de Wald, além de apresentarem boa capacidade preditiva, com o percentual de acertos maior que $90 \%$.

Para analisar a magnitude do efeito das variáveis explicativas sobre a ocupação nos setores, foram estimados os efeitos marginais. Os resultados indicam que a pandemia da COVID-19 impacta de forma significativa três dos quatro setores analisados: indústria, comércio e transporte. Especificamente, a presença da pandemia no $1^{\circ}$ trimestre de 2020 reduz a probabilidade de ocupação em 0,142 p.p. na indústria, 0,423 p.p. no setor de comércio e 0,054 p.p. no segmento de transporte.

É importante ressaltar, inicialmente, que os setores de comércio, indústria e serviços (incluindo o segmento de transportes) são aqueles que possuem maior participação, além da administração pública, na dinâmica do mercado de trabalho formal no Nordeste, tanto no período de relativo auge econômico, entre 2001 e 2010, quanto na fase de declínio, entre 2011 e 2016 (MACHADO, 2019). Isso comprova a importância de se analisar o impacto da COVID-19 sobre os referidos setores, que em função de sua relevância, podem sofrer perdas significativas desde o início da pandemia em função da entrada em vigor das medidas de enfrentamento à mesma.

Além disso, é importante analisar o panorama desses setores nos anos recentes para entender como o surgimento da pandemia pode ter impactado nesses grupamentos. Especificamente no caso da indústria, Silva Filho e Queiroz (2011) apontam que entre os anos de 1998 e 2008, houve crescimento expressivo no número de instalações na região nordestina, passando de 26.387 estabelecimentos para 85.007, com concomitante elevação na quantidade de empregos de 598.564 para 1.517.291. No entanto, o estudo de Machado (2019) denotou que o setor foi um dos primeiros a sofrer desaceleração econômica, com queda acentuada na criação de empregos formais a partir de 2010, passando a apresentar destruição líquida de postos de trabalhos a partir de 2015. Nesse sentido, o surgimento da pandemia da COVID-19 não apenas intensificou a dificuldade de recuperação do se- 
do Brasil

tor, mas também contribui para causar a falência de muitas empresas, o que determinou perdas significativas em termos de emprego.

Machado (2019) ainda afirma que no período 2001-2016, a região Nordeste apresentou taxa média de criação líquida de empregos de $6,83 \%$, sendo que o setor de Comércio teve participação de $1,18 \%$ a.a. neste total. A autora afirmou que o setor se manteve relativamente constante na variação de postos de trabalho no Nordeste até 2014. Todavia, a partir de 2015, momento de agravamento da crise econômica nacional, o setor começa a sofrer desaceleração, chegando a apresentar uma taxa de destruição líquida em 2016 da ordem de $-1,83 \%$. Assim, este setor, responsável pelo emprego de boa parcela da população nordestina, se vê diante de um novo desafio, a pandemia de COVID-19. Nesse sentido, verifica-se que já no início de seu enfrentamento é possível observar seus impactos negativos sobre o setor, dada a diminuição na probabilidade de ocupação no mesmo.

Em relação ao contexto anterior à pandemia no setor de transporte, Viana (2019) relatou que a região Nordeste apresenta defasagens em todos os seus principais modais de transporte, comparando-se a outras regiões do país, sendo que estas se agravam nos modais ferroviários, aéreo e hidroviário. Particularmente, este último apresentou o menor nível de investimento dentre os principais modais, apesar da região contar com uma vasta costa litorânea e rios longilíneos. As defasagens observadas e o baixo nível de investimentos tornam o setor de transporte vulnerável e com dificuldades de responder a crises, como a atual, proveniente da pandemia, o que é refletido pelo efeito marginal negativo para a variável da COVID-19. De qualquer forma, é importante mencionar que este setor apresenta o menor efeito marginal para esta variável, o que pode estar relacionado com a manutenção do transporte de insumos essenciais; como alimentos, produtos farmacêuticos e equipamentos de saúde; para o mercado nacional e exportação.

Por fim, o grupamento de construção, que não apresentou significância estatística para o efeito marginal da variável referente a COVID-19, é apontado por Machado (2019) como um dos setores que contribuíram de forma efetiva para a destruição líquida de empregos no Nordeste entre 2011 e 2016. Nesse sentido, é relevante salientar que o Brasil apresentou recessão econômica entre abril de 2014 e dezembro de 2016; e estagnação nos anos seguintes, como indica o Comitê de Datação de Ciclos Econômicos (CODACE). Como o nível de atividade do setor de construção é um indicativo do aquecimento ou desaquecimento da economia brasileira, espera-se que esse comportamento seja similar no caso da região Nordeste. Sendo assim, o modelo provavelmente não captou o efeito da variável proxy que representa a pandemia da COVID-19 sobre este setor pelo fato de o mesmo já estar passando por perdas em termos de ocupação e queda no nível de produção antes mesmo do advento da pandemia. Além disso, como a análise foi realizada para o $1^{\circ}$ trimestre de 2020, provavelmente não foi possível identificar com precisão o que seria efeito da conjuntura econômica e o que denotaria o impacto das medidas de enfrentamento ao novo coronavírus sobre o referido setor.

Com relação às demais variáveis de controle, o coeficiente do efeito marginal da va- 
riável que capta o gênero do indivíduo foi positivo e significativo para todos os setores, indicando que ser do gênero masculino aumenta a probabilidade de se estar ocupado nos quatro setores, sendo este aumento de 2,44 p.p. na indústria, 4,24 p.p. no comércio, 6,53 p.p. na construção e 3,30 p.p. no setor de transporte. Tal resultado corrobora com os dados da PNADC de 2018, onde verificou-se que a taxa de desemprego entre homens era de 11\%; já entre as mulheres de 14,2\%. De acordo com Pinheiro et al. (2009), as mulheres têm aumentado sua participação no mercado de trabalho, mas ainda estão inseridas de forma precária, uma vez que elas seguem mais representadas no trabalho doméstico, na produção para o próprio consumo e no trabalho não remunerado, enquanto os homens ocupam mais postos de trabalho com carteira assinada e de empregador. Adicionalmente, a partir de microdados da Pesquisa Nacional por Amostra de Domicílios (PNAD) 2013, Medeiros e Pinheiro (2018) observaram que o tempo ofertado de trabalho remunerado pelos homens é superior ao das mulheres. Nesse sentido, os homens respondem por $61,2 \%$ do total de horas trabalhadas, enquanto no caso do trabalho não remunerado ocorre o inverso, já que as mulheres representam $83 \%$ das referidas horas ofertadas, indicando certo grau de divisão sexual no mercado de trabalho.

A variável referente a cor apresentou efeito marginal significativo para os setores de comércio e construção. Ser branco ou amarelo aumenta a probabilidade de ocupação no

Tabela 2 - Resultados das estimativas do modelo Probit e seus respectivos efeitos marginais

\begin{tabular}{|c|c|c|c|c|c|c|c|c|}
\hline \multirow{2}{*}{ Variáveis } & \multicolumn{2}{|c|}{ Indústria } & \multicolumn{2}{|c|}{ Comércio } & \multicolumn{2}{|c|}{ Construção } & \multicolumn{2}{|c|}{ Transporte } \\
\hline & Coeficiente & $d y / d x$ & Coeficiente & $d y / d x$ & Coeficiente & dy/dx & Coeficiente & $d y / d x$ \\
\hline & $-0.01828^{*}$ & $-0.00142 * *$ & $-0.02799^{*}$ & $-0.00423 *$ & $-0.00127 \mathrm{~ns}$ & $-0.00003 \mathrm{~ns}$ & $-0.02576^{* *}$ & $-0.00054 * *$ \\
\hline COVID-19 & $-0,00784$ & $-0,00061$ & $-0,00604$ & $-0,0009$ & $-0,00899$ & $-0,00027$ & $-0,01088$ & $-0,00023$ \\
\hline & $0.30404 *$ & $0.02439 *$ & $0.27483^{*}$ & $0.04240^{*}$ & $1.49198^{*}$ & $0.06535^{*}$ & $1.15834 *$ & $0.03300^{*}$ \\
\hline Gênero & $-0,00671$ & $-0,00054$ & $-0,00507$ & $-0,00079$ & $-0,01588$ & $-0,06535$ & $-0,01455$ & $-0,00039$ \\
\hline & $0.00775 \mathrm{~ns}$ & $0.00061 \mathrm{~ns}$ & $0.06657^{*}$ & 0.01044* & $-0.10201 *$ & $-0.00291 *$ & $0.00179 \mathrm{~ns}$ & $0.00003 \mathrm{~ns}$ \\
\hline Cor & $-0,00772$ & $-0,00061$ & $-0,00583$ & $-0,00094$ & $-0,00948$ & $-0,00026$ & 0,01074 & $-0,00023$ \\
\hline & $0.00294 *$ & $0.00023 *$ & 0.00114* & $0.00017 *$ & $0.00080 *$ & $0.00002 *$ & 0.00933* & $0.00020^{*}$ \\
\hline Idade & $-0,00023$ & $-0,00002$ & $-0,00018$ & $-0,00003$ & $-0,00028$ & $-0,00001$ & $-0,00032$ & $-0,00001$ \\
\hline & $0.02138^{*}$ & $0.00168 *$ & $0.03536^{*}$ & $0.00540 *$ & $-0.01770^{*}$ & $-0.00053^{*}$ & $0.02881^{*}$ & $0.00062 *$ \\
\hline Anos de estudo & $-0,00076$ & $-0,00006$ & $-0,00058$ & $-0,00009$ & $-0,00087$ & $-0,0003$ & $-0,00101$ & $-0,00002$ \\
\hline & $0.15780^{*}$ & $0.01293 *$ & $0.12966 *$ & $0.02030 *$ & $0.10867 *$ & $0.00341 *$ & $0.10060^{*}$ & $0.00224 *$ \\
\hline Pessoa de Ref. & $-0,00747$ & $-0,00063$ & $-0,00574$ & $-0,00092$ & $-0,00888$ & $-0,00029$ & $-0,01043$ & $-0,00024$ \\
\hline & $0.23109^{*}$ & $0.01736 *$ & $.47453^{*}$ & $0.00092 *$ & $0.35452 *$ & $0.00341^{*}$ & $0.37569^{*}$ & $0.00741^{*}$ \\
\hline Urbano & $-0,00772$ & $-0,00055$ & $-0,00618$ & $-0,00079$ & $-0,00887$ & $-0,00029$ & $-0,01154$ & $-0,00023$ \\
\hline Constante & $\begin{array}{c}-2.43870^{*} \\
-0,01316\end{array}$ & & $\begin{array}{c}-2.21600 * \\
-0,01007\end{array}$ & & $\begin{array}{c}-3.11159^{*} \\
-0,01958\end{array}$ & & $\begin{array}{c}-3.83102 * \\
-0,02272\end{array}$ & \\
\hline
\end{tabular}

Fonte: Elaboração própria. Notas: Número de observações 512.272, Erros padrão entre parênteses, ${ }^{*} \mathrm{p}<0,01 ; * * \mathrm{p}<0,05 ; * * * \mathrm{p}<0,1 ;$ ns indica não significância. 
do Brasil

setor de comércio em 1,04 p.p., enquanto reduz as chances de ocupação na construção em 0,29 p.p. Tal resultado reflete o perfil ocupacional dos brasileiros ao realizar a segmentação por cor/raça. Dados do Retrato das Desigualdades de Gênero e Raça, elaborado pelo IPEA, indicam que no período compreendido entre 1995 e 2015, os indivíduos brancos possuíam, na média, nível de ocupação $18,46 \%$ maior que os não brancos no setor de comércio, enquanto os indivíduos não brancos apresentaram média 49,34\% mais elevada de ocupação no setor de construção, comparativamente aos brancos. Já nos setores de indústria e transporte, a cor/raça do indivíduo não é determinante para a ocupação na região Nordeste.

As estimativas indicam efeito marginal significativo e positivo para a variável idade, ou seja, um aumento de um ano de idade aumenta a probabilidade de estar ocupado em todos os setores analisados, sendo a magnitude de 0,023 p.p. na indústria, 0,017 no comércio, 0,0002 p.p. na construção e 0,02 no setor de transporte. Este resultado é corroborado pela literatura, como no trabalho de Cunha et. al (2011). Os autores apontam que o desemprego no Brasil é um fenômeno que atinge principalmente os jovens em função da concorrência com indivíduos mais velhos, que tendem a possuir maior experiência profissional. Os resultados também demonstram que a escolaridade é um importante determinante da probabilidade de ocupação, apresentando efeito marginal positivo e significativo em três dos setores analisados. Nesse sentido, o aumento de um ano de estudo aumenta a probabilidade de que os indivíduos estejam ocupados em 0,168 p.p. no setor de indústria, 0,54 p.p. no comércio e 0,062 p.p. no segmento de transporte. Este resultado também é encontrado por Seabra (2002) ao analisar a influência da escolaridade sobre os salários e sobre a empregabilidade na região metropolitana do Rio de Janeiro. O autor observou que com o processo de globalização, a capacitação é um dos fatores mais importantes para a sobrevivência das empresas. Por outro lado, no caso do setor de construção, o aumento de um ano de estudo reduz as chances de emprego em 0,053 p.p., o que pode ser explicado pelos resultados da pesquisa de Cantisani e Castelo (2015). Os autores observaram que quase $70 \%$ dos trabalhadores no setor haviam interrompido os estudos no ensino fundamental completo ou inferior. Além disso, a média de anos de estudo entre os trabalhadores da construção é $23 \%$ menor que a média do país.

Ser a pessoa de referência no domicílio aumenta a probabilidade de ocupação nos quatros setores analisados da região Nordeste. A magnitude dessa influência é de 1,3 p.p. na indústria, 2,03 p.p. no comércio, 0,34 p.p. no comércio e 0,22 p.p. no transporte. Este resultado vai ao encontro daquele encontrado por Arruda et al. (2017), onde verificase que os chefes de família possuem, em média, probabilidade $4 \%$ menor de estarem desempregados, quando comparados aos não chefes.

Por fim, residir no meio urbano tem efeito positivo e significativo nas chances de que os indivíduos estejam ocupados em todos os setores analisados. Especificamente, aumenta a probabilidade de ocupação na indústria em 1,74 p.p., 0,1 p.p. no comércio, 0,34 p.p. no setor de construção e 0,74 p.p. no de transporte. Este resultado está relacionado 
ao fato de os setores estudados concentrarem suas atividades no referido meio.

\subsection{Evolução da ocupação por setores e subsetores da região nordestina}

Esta subseção tem como objetivo apresentar, de forma mais pormenorizada, a evolução da ocupação ao longo dos primeiros trimestres durante o período 2017-2020 nos quatro setores analisados, bem como nos subsetores que compõem tais agregados. A PNADC realiza um agrupamento das atividades econômicas especificadas na Classificação Nacional de Atividades Econômicas Domiciliar para efeito de divulgação. Assim, por meio dos dados desagregados, será possível analisar a dinâmica da ocupação dentro dos setores, bem como verificar entre aqueles indicados pelo modelo econométrico como afetados pela pandemia da COVID-19, quais foram mais impactados.

A Tabela 3 trata da evolução da ocupação no período analisado para o setor industrial e seus respectivos subsetores. É possível observar que o referido setor vinha passando por queda no nível de ocupação desde o $1^{\circ}$ trimestre de 2018, comparando-se ao mesmo período do ano anterior, o que está atrelado a estagnação econômica verificada no país, particularmente no período 2014-2016. É possível ainda perceber que a magnitude desse decrescimento aumentou consideravelmente quando se compara o $1^{\circ}$ trimestre de 2020 em relação ao mesmo período em 2019, com variação negativa de $15,66 \%$, o que corrobora os resultados obtidos através do modelo econométrico, ou seja, a pandemia acentua as dificuldades já enfrentadas por este setor no período de recessão e estagnação econômica, causando ainda mais perdas de postos de trabalho.

Desagregando-se o setor, verifica-se que todos os quatro subsetores que o compõem sofrem significativa redução no nível de ocupação quando se compara o $1^{\circ}$ trimestre de 2020 com o mesmo período em 2019. A indústria extrativa e o subsetor de água, esgoto, atividades de gestão de resíduos e descontaminação são os que apresentam as maiores perdas nesse período. No caso da indústria extrativa, é interessante notar que há queda de 2,55\% no nível de ocupação entre o $1^{\circ}$ trimestre de 2017 e o $1^{\circ}$ trimestre de 2018 , mas que no período seguinte, entre 2018 e 2019, o subsetor apresenta elevação de 8,73\%, mas volta a cair, de forma bastante expressiva, no período compreendido entre 2019 e 2020 , com magnitude igual a 23,29\%. Por sua vez, a indústria de transformação saiu de uma queda de $0,88 \%$ no $1^{\circ}$ trimestre de 2018 relativamente ao $1^{\circ}$ trimestre de 2019 , para uma redução de 14,75\% no período posterior (2019 a 2020), o que indica que esse subsetor foi bastante afetado já no início do enfrentamento à COVID-19. Por sua vez, o segmento de água, esgoto, atividades de gestão de resíduos e descontaminação apresenta perda acentuada no primeiro período analisado, de 20,27\% na ocupação, passando por um momento de recuperação entre 2018 e 2019, com variação positiva de $0,56 \%$, e a seguir, quando se compara 2020 em relação à 2019, o subsetor apresenta queda expressiva de $25 \%$.

O setor de comércio também já vinha apresentando queda na ocupação desde o $1^{\circ}$ 

do Brasil

Tabela 3 - Evolução da ocupação no setor industrial

\begin{tabular}{|c|c|c|}
\hline Setor & Período & Var. \% \\
\hline \multirow{3}{*}{ Indústria } & $1^{\circ}$ tri. $2019-1^{\circ}$ tri. 2020 & $-15.66 \%$ \\
\hline & $1^{\circ}$ tri. $2018-1^{\circ}$ tri. 2019 & $-0.45 \%$ \\
\hline & $1^{\circ}$ tri. $2017-1^{\circ}$ tri. 2018 & $-2.24 \%$ \\
\hline Subsetor & Período & Var. \% \\
\hline \multirow{3}{*}{ Indústria Extrativa } & $1^{\circ}$ tri. $2019-1^{\circ}$ tri. 2020 & $-23.29 \%$ \\
\hline & $1^{\circ}$ tri. $2018-1^{\circ}$ tri. 2019 & $8.73 \%$ \\
\hline & $1^{\circ}$ tri. $2017-1^{\circ}$ tri. 2018 & $-2.55 \%$ \\
\hline \multirow{3}{*}{ Indústria de Transformação } & $1^{\circ}$ tri. $2019-1^{\circ}$ tri. 2020 & $-14.75 \%$ \\
\hline & $1^{\circ}$ tri. $2018-1^{\circ}$ tri. 2019 & $-0.88 \%$ \\
\hline & $1^{\circ}$ tri. $2017-1^{\circ}$ tri. 2018 & $-0.15 \%$ \\
\hline \multirow{3}{*}{ Eletricidade e Gás } & $1^{\circ}$ tri. $2019-1^{\circ}$ tri. 2020 & $-6.35 \%$ \\
\hline & $1^{\circ}$ tri. $2018-1^{\circ}$ tri. 2019 & $-4.55 \%$ \\
\hline & $1^{\circ}$ tri. $2017-1^{\circ}$ tri. 2018 & $-11.41 \%$ \\
\hline \multirow{3}{*}{ Água, Esgoto, Atividades de gestão de resíduos e Descontaminação } & $1^{\circ}$ tri. $2019-1^{\circ}$ tri. 2020 & $-25.00 \%$ \\
\hline & $1^{\circ}$ tri. $2018-1^{\circ}$ tri. 2019 & $0.56 \%$ \\
\hline & $1^{\circ}$ tri. $2017-1^{\circ}$ tri. 2018 & $-20.27 \%$ \\
\hline
\end{tabular}

Fonte: Elaboração própria.

trimestre de 2018, como indica a Tabela 4, o que está relacionado com o baixo nível de atividade econômica do país neste período, porém nota-se que a queda na ocupação se acentua significativamente no $1^{\circ}$ trimestre de 2020, comparativamente ao mesmo período do ano anterior, o que também corrobora os resultados do modelo econométrico estimado.

Analisando-se os dois subsetores que são agregados em Comércio, observa-se que ambos apresentam queda acentuada no período entre 2019 e 2020 e a magnitude é semelhante, representando $17,58 \%$ de queda no subsetor de comércio e reparação de veículos automotores e motocicletas, e 15,02\% no de comércio, exceto de veículos automotores e motocicletas. Quando se observa os períodos anteriores, percebe-se que ambos os segmentos já vinham sofrendo queda no nível de ocupação desde 2018, mas em proporção muito menor, o que parece indicar que não só o contexto econômico está influenciando a atividade destes setores, mas também a pandemia e as medidas adotadas de combate à mesma.

Econômica-Niterói, v. 22, n. 1, p. 9-30. Junho, 2020 
Tabela 4 - Evolução da ocupação no setor comercial

\begin{tabular}{l|l|l}
\hline Setor & Período & Var. \% \\
\hline Comércio & $1^{\circ}$ tri. $2019-1^{\circ}$ tri. 2020 & $-15.37 \%$ \\
& $1^{\circ}$ tri.2018 $-1^{\circ}$ tri. 2019 & $-1.62 \%$ \\
\hline Subsetor & $1^{\circ}$ tri. $2017-1^{\circ}$ tri. 2018 & $-2.14 \%$ \\
\hline & Período & Var. \% \\
\hline Comércio e reparação de veículos automotores e motocicletas & $1^{\circ}$ tri. $2019-1^{\circ}$ tri. 2020 & $-17.58 \%$ \\
& $1^{\circ}$ tri.2018 $-1^{\circ}$ tri. 2019 & $-3.53 \%$ \\
\hline & $1^{\circ}$ tri. $2017-1^{\circ}$ tri. 2018 & $-1.16 \%$ \\
\hline Comércio, exceto de veículos automotores e motocicletas & $1^{\circ}$ tri. $2019-1^{\circ}$ tri. 2020 & $-15.02 \%$ \\
& $1^{\circ}$ tri.2018 $-1^{\circ}$ tri. 2019 & $-1.31 \%$ height \\
\hline
\end{tabular}

Fonte: Elaboração própria.

Em seguida, tem-se a análise desagregada da evolução da ocupação no setor de construção, vide Tabela 5, abaixo. É importante frisar que o modelo econométrico não captou significância estatística da variável utilizada como proxy para a pandemia da COVID19 sobre a ocupação neste grupamento. É possível observar que desde 2017 o setor de construção passava por um significativo decrescimento em termos de ocupação, apesar do referido decrescimento ter sido amenizado entre 2018 e 2019, passando de 11,78\% para $1,96 \%$. Entretanto, no $1^{\circ}$ trimestre de 2020 , o setor voltou a apresentar queda significativa em termos de ocupação, comparativamente ao mesmo período do ano anterior. É importante ressaltar que a dinâmica do setor de construção representa uma espécie de 'termômetro' do nível de atividade econômica no Brasil. Nesse sentido, o Comitê de Datação de Ciclos Econômicos (CODACE), associado à Fundação Getúlio Vargas (FGV), apontou que a economia brasileira passava por um processo de estagnação desde fim de 2016 e que a partir do $1^{\circ}$ trimestre de 2020 entra em um quadro recessivo. Logo, o setor de construção na região Nordeste parece acompanhar a movimentação da economia brasileira. Dentre os três subsetores, é possível observar a mesma tendência geral: um significativo decrescimento entre 2017 e 2018, passando por uma recuperação entre 2018 e 2019, chegando a apresentar aumento da ocupação nos subsetores de Obras de infraestrutura e Serviços especializados para construção, com retração expressiva no $1^{\circ}$ trimestre de 2020 comparativamente ao $1^{\circ}$ trimestre de 2019.

Por fim, tem-se a evolução da ocupação no setor de transporte. Este setor foi o único entre os três analisados que apresentou uma inversão de tendência. Enquanto indústria e comércio já vinham sofrendo perdas ocupacionais e a pandemia acentua esse movimento, 
Tabela 5 - Evolução da ocupação no setor de construção

\begin{tabular}{l|l|l}
\hline Setor & Período & Var. \% \\
\hline Construção & $1^{\circ}$ tri. $2019-1^{\circ}$ tri. 2020 & $-11.32 \%$ \\
& $1^{\circ}$ tri.2018 $-1^{\circ}$ tri. 2019 & $-1.96 \%$ \\
\hline Subsetor & $1^{\circ}$ tri. $2017-1^{\circ}$ tri. 2018 & $-11.78 \%$ \\
\hline & Período & Var. \% \\
\hline Construção e incorporação de edifícios & $1^{\circ}$ tri. $2019-1^{\circ}$ tri. 2020 & $-11.36 \%$ \\
\hline & $1^{\circ}$ tri.2018 $-1^{\circ}$ tri. 2019 & $-2.86 \%$ \\
\hline Obras de infraestrutura & $1^{\circ}$ tri. $2017-1^{\circ}$ tri. 2018 & $-10.45 \%$ \\
\hline & $1^{\circ}$ tri. $2019-1^{\circ}$ tri. 2020 & $-2.29 \%$ \\
& $1^{\circ}$ tri.2018 $-1^{\circ}$ tri. 2019 & $0.99 \%$ \\
\hline & $1^{\circ}$ tri. $2017-1^{\circ}$ tri. 2018 & $-3.19 \%$ \\
\hline
\end{tabular}

Fonte: Elaboração própria.

o setor de transporte passava por uma recuperação entre o $1^{\circ}$ trimestre de 2018 e o $1^{\circ}$ trimestre de 2019, da ordem de 4,38\%, como indica a Tabela 6. Assim, este setor passou de uma situação de recuperação para forte retração na ocupação, apresentando uma queda de $18,05 \%$ entre o $1^{\circ}$ trimestre de 2019 e o $1^{\circ}$ trimestre de 2020 , o que demonstra a efeito da pandemia e das medidas de enfrentamento.

Desagregando-se este setor nos cinco subsetores que o compõem, constata-se que houve queda muito expressiva nos três modais de transporte: transporte terrestre, aquaviário e aéreo. No caso do transporte terrestre, no $1^{\circ}$ trimestre de 2020 , tem-se queda de $20,21 \%$ em relação ao mesmo período de 2019 e verifica-se que o mesmo havia apresentado leve aumento de 4,20\% no nível de ocupação entre 2018 e 2019. O segmento de transporte aquaviário é o mais impactado, com redução de $42,11 \%$ entre o $1^{\circ}$ trimestre de 2019 e 2020, sendo que o mesmo já passava por decréscimo desde 2017, mas em 2020 a magnitude da redução foi bem mais acentuada.

O subsetor seguinte, transporte Aéreo, não apresentou variação entre os períodos compreendidos entre 2017 a 2018 e 2018 a 2019, mas no ano de 2020 sofreu queda de 26,67\% em seu nível de ocupação. Esse movimento de decrescimento em termos de ocupação no transporte aéreo pode indicar queda do nível de atividade do turismo na região, uma vez que com as medidas de enfretamento à pandemia da COVID-19, observa-se redução da demanda por voos, bem como restrições sobre as frotas aéreas e entrada de não residentes 
Tabela 6 - Evolução da ocupação no setor de transporte

\begin{tabular}{|c|c|c|}
\hline Setor & Período & Var.\% \\
\hline \multirow{3}{*}{ Transporte } & $1^{\circ}$ tri. $2019-1^{\circ}$ tri. 2020 & $-18.05 \%$ \\
\hline & $1^{\circ}$ tri. $2018-1^{\circ}$ tri. 2019 & $4.38 \%$ \\
\hline & $1^{\circ}$ tri. $2017-1^{\circ}$ tri. 2018 & $-5.05 \%$ \\
\hline Subsetor & Período & Var.\% \\
\hline \multirow{3}{*}{ Transporte Terrestre } & $1^{\circ}$ tri. $2019-1^{\circ}$ tri. 2020 & $-20.21 \%$ \\
\hline & $1^{\circ}$ tri. $2018-1^{\circ}$ tri. 2019 & $4.20 \%$ \\
\hline & $1^{\circ}$ tri. $2017-1^{\circ}$ tri. 2018 & $-5.29 \%$ \\
\hline \multirow{3}{*}{ Transporte Aquaviário } & $1^{\circ}$ tri. $2019-1^{\circ}$ tri. 2020 & $-42.11 \%$ \\
\hline & $1^{\circ}$ tri. $2018-1^{\circ}$ tri. 2019 & $-5.00 \%$ \\
\hline & $1^{\circ}$ tri. $2017-1^{\circ}$ tri. 2018 & $-14.89 \%$ \\
\hline \multirow{3}{*}{ Transporte Aéreo } & $1^{\circ}$ tri. $2019-1^{\circ}$ tri. 2020 & $-26.67 \%$ \\
\hline & $1^{\circ}$ tri. $2018-1^{\circ}$ tri. 2019 & $0.00 \%$ \\
\hline & $1^{\circ}$ tri. $2017-1^{\circ}$ tri. 2018 & $0.00 \%$ \\
\hline \multirow{3}{*}{ Armazenamento e Atividades auxiliares do Transporte } & $1^{\circ}$ tri. $2019-1^{\circ}$ tri. 2020 & $4.86 \%$ \\
\hline & $1^{\circ}$ tri. $2018-1^{\circ}$ tri. 2019 & $-2.70 \%$ \\
\hline & $1^{\circ}$ tri. $2017-1^{\circ}$ tri. 2018 & $-12.94 \%$ \\
\hline \multirow{3}{*}{ Correio e outras atividades de entrega } & $1^{\circ}$ tri. $2019-1^{\circ}$ tri. 2020 & $5.00 \%$ \\
\hline & $1^{\mathrm{o}}$ tri. $2018-1^{\mathrm{o}}$ tri. 2019 & $23.71 \%$ \\
\hline & $1^{\circ}$ tri. $2017-1^{\circ}$ tri. 2018 & $24.36 \%$ \\
\hline
\end{tabular}

Fonte: Elaboração própria. 
do Brasil

em localidades da região tipicamente conhecidas como pontos turísticos.

\title{
5 Considerações finais
}

O presente estudo teve como objetivo analisar a influência da pandemia da COVID-19 sobre os níveis de ocupação nos setores da indústria, comércio, construção e transporte na região Nordeste do Brasil, uma região potencialmente impactada pela crise com maior severidade devido aos seus níveis mais elevados de vulnerabilidade socioeconômica com relação ao restante do país. Para tal, foram estimados quatro modelos Probit para cada um dos setores.

De acordo com os resultados, dos quatro setores analisados, apenas o de construção não foi afetado, sendo os demais impactados negativamente pela pandemia da COVID19, implicando em redução dos níveis de ocupação no $1^{\circ}$ trimestre de 2020 em relação aos demais anos analisados.

Tais resultados são fundamentais para a elaboração de políticas públicas de retomada econômica no período pós pandemia no Nordeste do país, uma vez que os setores produtivos mais afetados pela crise deverão ser aqueles para onde serão dirigidos maiores volumes de recursos, com o intuito de promover o crescimento econômico de maneira mais eficiente em um futuro próximo.

Por fim, é necessário destacar que o presente estudo apresenta limitações. Os dados utilizados para estudar a magnitude do impacto da crise da COVID-19 sobre os níveis de ocupação nos setores produtivos considerados são referentes ao $1^{\circ}$ trimestre de 2020 . Como o primeiro caso oficial da COVID-19 foi registrado no dia 26 de fevereiro de 2020 e as primeiras medidas do governo federal para administrar a crise foram caracterizadas como tardias, é possível imaginar que as informações coletadas não sejam capazes de refletir com fidedignidade a magnitude real do impacto da pandemia sobre os níveis de ocupação na região Nordeste. Nesse sentido, um maior horizonte temporal seria capaz de capturar melhor a influência da pandemia da COVID-19 sobre a ocupação no mercado de trabalho para a referida região.

\section{COVID-19 pandemic and occupation in the labor market: the case the Northeast of Brazil}

\begin{abstract}
The main objective of this work is to analyze the impact of the COVID-19 pandemic on occupancy levels in the sectors of industry, commerce, construction and transportation in the Northeast region of Brazil through the estimation of four Probit models. With the exception of the construction sector, all other sectors considered were negatively impacted by the pandemic. Specifically, the
\end{abstract}

Econômica-Niterói, v. 22, n. 1, p. 9-30. Junho, 2020 
presence of COVID-19 in the 1st quarter of 2020 reduced the likelihood of occupation by 0.142 p.p. in industry, 0.423 p.p. in commerce and 0.054 p.p. in the transportation segment.

Keywords: COVID-19, Employment, Brazilian Northeast.

JEL: J01, J00

\section{Referências bibliográficas}

ABRAMO, L. Desigualdades de Gênero e Raça no Mercado de Trabalho Brasileiro. Ciência e Cultura, São Paulo, v.58, n.4, p. 40-41, dez. 2006.

AÇIKGÖZ, Ö.; GÜNAY, A. The early impact of the Covid-19 pandemic on the global and Turkish economy. Turkish Journal of Medical Sciences, Ankara, v. 50, n. 1, p. 520-526, abril. 2020.

ALVES, E. L. G.; SOARES, F. V. Ocupação e escolaridade: tendências recentes na Grande São Paulo. Brasília: Ipea, 1996. (Texto para Discussão, n. 428).

ARRUDA, E. F.; GUIMARÃES, D. B.; CASTELAR, I. Uma Análise do desemprego severo nas regiões Sul e Sudeste do Brasil em 2013. Planejamento e Políticas Públicas, n. 48, p. 207-228, jan/jun. 2017.

ARULAMPALAM, W. A note on estimated coefficients in random effects probit models. Oxford Bulletin of Economics and Statistics, v. 61, n. 4, p. 597-602, 1999.

BORGES, Â. Impactos do desemprego e da precarização sobre famílias metropolitanas. Revista Brasileira de Estudos de População, São Paulo, v. 23, n. 2, p. 205-222, jul./dez. 2006.

CANTISANI, A. F.; CASTELO, A. M. O perfil dos trabalhadores da construção civil. Conjuntura da Construção, v.13, n.1, p.10-13, mar. 2015.

COATES, B. et al. Shutdown: estimating the COVID-19 employment shock. Grattan Institute Working Paper, n.3, p. 2-59, april, 2020.

CODEÇO, C. T. et al. Estimativa de risco de espalhamento da COVID-19 no Brasil e avaliação da vulnerabilidade socioeconômica nas microrregiões brasileiras. Rio de Janeiro: FIOCRUZ/PROCC, mar. 2020.

COMISIÓN ECONÓMICA PARA AMÉRICA LATINA Y EL CARIBE (CEPAL). 

do Brasil

América Latina y el Caribe ante la pandemia del COVID-19: Efectos económicos y sociales. Informe Especial COVID-19, n.1, p.1-15, abr. 2020.

COMITÊ CIENTÍFICO - CONSÓRCIO NORDESTE SUBCOMITÊ DE EPIDEMIOLOGIA, Pandemia da Covid-19 no Nordeste do Brasil: Situação Atual e Recomendações, 2020.

CUNHA, D.; DE ARAÚJO, A. A.; DE LIMA, J. E. Determinantes do desemprego e inatividade de jovens no Brasil metropolitano. Revista de Economia e Agronegócio, v. 9, n. 3, p.369-392, nov. 2011.

DAVIDSON, R.; MACKINNON, J. G. Econometric theory and methods. New York: Oxford University Press, 2004.

DEV, S. M.; SENGUPTA, R. Covid-19: Impact on the Indian economy. Indira Gandhi Institute of Development Research, Mumbai, n.13, p. 1-48, apr. 2020.

FERNANDES, R.; PICCHETTI P. Uma análise da estrutura do desemprego e da inatividade no Brasil metropolitano. Pesquisa e Planejamento Econômico, Rio de Janeiro, v. 29, n.1. p. 87-112, abr. 1999.

FIGUEIREDO, A. M. R.; MARTA, J. M. C.; GUIMARÃES, P. Determinantes da ocupação do núcleo familiar composto na área rural do Brasil. Economia e Desenvolvimento, n. 22, 2010.

FLEMING, C. M.; KLER, P. I'm too clever for this job: a bivariate probit analysis on overeducation and job satisfaction in Australia. Applied Economics, v. 40, n.9, p. 1123-1138, fev. 2008.

FUNDAÇÃO GETÚllO VARGAS (FGV). Comitê de Datação de Ciclos Econômicos. Disponível em: <https://portalibre.fgv.br/>. Acesso em: 21 jun. 2018.

GANGOPADHYAYA, A.; GARRETT, A. B. Unemployment, Health Insurance, and the COVID-19 Recession. Urban Institute. Timely Analysis of Immediate Health Policy Issues, p. 1-8, apr. 2020.

GUIMARÃES, P. W.; DOS SANTOS, C. M. Determinantes da ocupação no mercado de trabalho de maridos e esposas. Revista Brasileira de Gestão e Desenvolvimento Regional, Taubaté, v. 6, n. 2, p.23-43, mai/ago. 2010.

HEVIA, C.; NEUMEYER, A. A Conceptual Framework for Analyzing the Economic Impact of COVID-19 and its Policy Implications. UNDP LAC COVID-19 Policy Documents Series. v. 1, p. 29, 2020.

INSTITUTO BRASILEIRO DE GEOGRAFIA E ESTATÍSTICA (IBGE). Indicadores IBGE - Pesquisa Mensal de Serviços. mar. 2020.

PINHEIRO, L. et al. Retrato das Desigualdades de Gênero e Raça - 1995 a 2015. Brasília, DF: IPEA, 2017.

INTERNATIONAL LABOUR ORGANIZATION (ILO). COVID-19 and the world of 
work: Impact and policy responses. Geneva: ILO Monitor 1st Edition. mar. 2020.

INTERNATIONAL MONETARY FUND (IMF). World Economic Outlook, April 2020: The Great Lockdown. Washington: World Economic Outlook Reports. abr. de 2020.

KINGDON, G.; KNIGHT, J. The incidence of unemployment in South Africa. University of Oxford: Oxford, 2000.

LAING, T. The economic impact of the Coronavirus 2019 (Covid-2019): Implications for the mining industry. The Extractive Industries and Society, vol. 7,2 p. 580-582, apr. 2020.

MACHADO, B. L. Dinâmica do mercado de trabalho formal no Nordeste: criação e destruição de empregos de 2001 a 2016. In: IX Jornada Internacional de Políticas Públicas, São Luís - Maranhão, 2019.

MAKRIDIS, C. A.; HARTLEY, J. S. The Cost of COVID-19: A Rough Estimate of the 2020 US GDP Impact. Mercatus Center at George Mason University: Policy Brief Special Edition. apr. 2020.

MEDEIROS, M.; PINHEIRO, L. S. Desigualdades de gênero em tempo de trabalho pago e não pago no Brasil, 2013. Brasília: Revista Sociedade e Estado, v. 33, n. 1, p. 161-187. Jan/abr. 2018.

MONTE, P. A.; PENIDO, M. R. J. Determinantes da duração esperada do emprego urbano e rural no Nordeste brasileiro. Brasília: Revista de Economia e Sociologia Rural, v. 46, n. 4, p. 989-1013, 2008.

PINHEIRO, L. et al. Retratos das Desigualdades de Gênero e Raça - $3^{\text {a }}$ Edição. Brasília: Ipea: SPM: UNIFEM, p. 1-36, 2009.

SEABRA, A. A. de. Escolaridade, salários e empregabilidade: implicações no mercado de empregos do Rio de Janeiro. Dissertação (Gestão Empresarial) - Escola Brasileira de Administração Pública e de Empresas, Fundação Getúlio Vargas (FGV). Rio de Janeiro, p.58. 2002.

SILVA FILHO, L. A. da; QUEIROZ, S. N. de. Recuperação econômica e emprego formal: avaliação para o Nordeste brasileiro entre 2000 e 2008. São Leopoldo: Perspectiva Econômica, v.7 (1), p. 42-54, jan/jun. 2011.

SILVA, P. L. do N.; PESSOA, D. G. C.; LILA, M. F. Análise estatística de dados da PNAD: incorporando a estrutura do plano amostral. Rio de Janeiro: Ciência e Saúde Coletiva, v. 7, p. 659-670, jan. 2002.

SPURK, D.; STRAUB, C. Flexible employment relationships and careers in times of the COVID-19 pandemic. Journal of Vocational Behavior, v. 119, jun. 2020.

VIANA, F. L. E. Panorama da infraestrutura no Nordeste do Brasil: transportes. Escritório técnico de estudos econômicos do Nordeste - ETENE. v. 4. n. 63, jan. 2019. 
30 Pandemia da COVID-19 e ocupação no mercado de trabalho: o caso da região Nordeste do Brasil

WAGNER, A. F. What the stock market tells us about the post-COVID-19 world. Nature Human Behaviour, v. 4, n. 5, p. 440-440, apr. 2020.

YAMIN, M. Counting the cost of COVID-19. International Journal of Information Technology. p. 1-7, may. 2020.

Recebido em 25 de junho de 2020.

Aceito para publicação em 01 de setembro de 2020.

Econômica-Niterói, v. 22, n. 1, p. 9-30. Junho, 2020 